\title{
A trajetória histórica do programa interunidades de doutoramento em enfermagem e sua contribuição para a enfermagem brasileira
}

\author{
THE HISTORY OF THE NURSING INTERUNITS DOCTORAL PROGRAM AND \\ ITS CONTRIBUTION TO BRAZILIAN NURSING \\ TRAYECTORIAHISTÓRICA DEL PROGRAMA INTERUNIDADES DE DOCTORADO EN \\ ENFERMERÍA Y SU CONTRIBUCIÓN PARA LA ENFERMERÍA BRASILEÑA
}

\author{
Maria das Graças Carvalho Ferriani ${ }^{1}$, Isília Aparecida Silva ${ }^{2}$, Emília Campos de Carvalho $^{3}$
}

\author{
1 Professora Titular do \\ Departamento de \\ Enfermagem Materno- \\ Infantil e Saúde Pública da \\ Escola de Enfermagem de \\ Ribeirão Preto da \\ Universidade de São Paulo \\ (EERP/USP). \\ caroline@eerp.usp.br \\ 2 Professora Titular do \\ Departamento de \\ Enfermagem Materno- \\ Infantil e Psiquiátrica da \\ EE/USP. isasilva@usp.br \\ 3 Professora Titular do \\ Departamento de \\ Enfermagem geral e \\ Especializada da EERP/USP.
}

\begin{abstract}
RESUMO
Objetivou-se descrever a trajetória histórica do Programa Interunidades de Doutoramento em Enfermagem nos campi de São Paulo e de Ribeirão Preto, no período de 1981 a 2004. Coletou-se dados através de documentos existentes do Programa (resoluções, pareceres, as fichas do alunos matriculados, relatórios, dentre outros). O Programa Interunidades de Doutoramento em Enfermagem foi aprovado pela Câmara de PósGraduação da Universidade de São Paulo em 25 de maio de 1981. Durante muitos anos, até por volta do início da década de 1990, a demanda principal de candidatos era das próprias Unidades responsáveis pelo curso, seguida de professores de outras Escolas de Enfermagem do Brasil. O Programa Interunidades tem formado pesquisadores para diversas Universidades brasileiras e da América do Sul, que formam e lideram células de pesquisa, dando origem a novos programas de pósgraduação.
\end{abstract}

\section{DESCRITORES}

Educação de pós-graduação em enfermagem (história).

Docentes.

História da enfermagem.

\begin{abstract}
Authors aimed at describing the history of the Nursing Interunits Doctoral Program offered at the São Paulo and Ribeirão Preto campi from 1981 to 2004. Data were collected based on the Program documents (resolutions, opinion, students' records, reports, etc). The Nursing Interunits Doctoral Program was approved by the University of São Paulo Graduate Council in May 25, 1981. During the past several years, until the beginning of the 1990s, the demand was centered in the students from the Schools responsible for the Program, followed by faculty from other Nursing Schools in Brazil. The Program has resulted in the formation of researchers from different Brazilian and South American Universities, leading the creation of research groups and originating new graduate programs.
\end{abstract}

\section{KEY WORDS}

Education, nursing, graduate

(history).

Faculty.

History of nursing.

\section{RESUMEN}

En este estudio se tuvo como objetivo describir la trayectoria histórica del Programa Interunidades de Doctorado en Enfermería en los campus de São Paulo y de Ribeirão Preto, en el período de 1981 a 2004. Los datos fueron recolectados a través de documentos existentes del Programa (resoluciones, constancias, fichas de alumnos matriculados, informes, entre otros). El Programa Interunidades de Doctorado en Enfermería fue aprobado por la Cámara de Post Grado de la Universidad de São Paulo el 25 de mayo de 1981. Durante muchos años, hasta el inicio de la década de 1990, la demanda principal de candidatos era de las propias Unidades responsables del curso, seguida de profesores de otras Escuelas de Enfermería de Brasil. El Programa Interunidades ha formado investigadores para diversas Universidades brasileñas y de América del Sur, que forman y lideran células de investigación, dando origen a nuevos programas de Post Grado.

\section{DESCRIPTORES}

Educación de Post Grado en enfermería (historia).

Docentes.

Historia de la enfermería. 
As Escolas de Enfermagem da Universidade de São Paulo, nos campi de São Paulo (EE) e Ribeirão Preto (EERP), ao criarem seus primeiros cursos de mestrados em 1973 e 1975, respectivamente, já vislumbravam para futuro próximo a instalação do curso em nível de doutorado.

Em 1979, as duas Escolas apresentaram à Câmara de Pós-Graduação da Universidade de São Paulo suas propostas para criação de seus doutorados, as quais foram analisadas pela Câmara de Pós-Graduação da USP. O parecer final foi de que não havia massa crítica para as referidas Unidades oferecerem, isoladamente, seus cursos de doutoramento, recomendando a organização de um curso único envolvendo as duas Escolas.

Receberam tal incumbência, as Professoras Doutoras Wanda de Aguiar Horta, Amália Correia de Carvalho, Evalda Cançado Arantes, docentes da EE e Maria Aparecida Minzoni, Maria Helena Machado e Nilza Tereza Rotter Pelá, docentes da EERP.

Aos 15 de abril de 1981, foi encaminhada à Câmara de Pós-Graduação a proposta do Programa Interunidades de Doutoramento em Enfermagem, juntamente com um ofício assinado pelos diretores da EE e EERP, respectivamente, os professores Doutores Carlos da Silva Lacaz e Antonio Martinez, como segue:

\section{"Senhor Coordenador,}

As Comissões de Pós-Graduação das Escolas de Enfermagem da Universidade de São Paulo, de São Paulo (EE) e Ribeirão Preto (EERP), solicitam a autorização dessa Câmara para a implantação de um programa conjunto de pósgraduação, nível de doutorado.

A pós-graduação em enfermagem a nível de doutorado é necessidade urgente na Universidade de São Paulo. Ambas as Unidades, tanto a do Campus de São Paulo quanto a de Ribeirão Preto, carecem de um programa que facilite o desenvolvimento e o aperfeiçoamento de seu corpo docente e que propicie a seus membros os meios para a ascensão na carreira universitária. A Escola de Enfermagem, de São Paulo, já conta com grande experiência no campo de pós-graduação. De 1959 a 1970, antes da reforma universitária, portanto, manteve um curso para preparo de docente e chefes de serviços de enfermagem que beneficiou enfermeiros de todo Brasil, Portugal, de países da América Latina e da África, estes últimos através de bolsas de estudos e incentivos oferecidos pela OPAS/ OMS. Em 1973 teve início o programa, nível de mestrado, pelo qual foram tituladas com o grau de Mestre 52 enfermeiras.
A Escola de Enfermagem de Ribeirão Preto iniciou a pós-graduação em 1967, com um curso denominado "Bases de Experimentação em Enfermagem", destinado a aperfeiçoar os docentes no campo da investigação científica. Em 1975 implantou o programa de pós-graduação a nível de Mestrado na área de Enfermagem Psiquiátrica pelo qual já foram titulados 18 profissionais. Curso de mesmo nível foi iniciado em 1979 na Área Enfermagem Geral e que conta atualmente com 11 alunos.

O programa para cuja implantação está sendo solicitada autorização dessa Câmara foi planejado em conjunto, pelas duas Unidades, com o intuito de somar esforços visando ao aproveitamento máximo das respectivas potencialidades em termos de corpo docente, facilidades e condições para a pesquisa no campo da enfermagem. A inexistência de outros cursos de pós-graduação em enfermagem em nível de doutorado no País torna imprescindível e urgente a introdução de, pelo menos, um deles na USP, a fim de atender as necessidades e aspirações dos docentes das duas Unidades de Enfermagem e das demais Escolas congêneres das universidades governamentais ou privadas do Brasil. A matéria de que cuida o presente processo é de mais alta relevância para o desenvolvimento da enfermagem não apenas no Estado de São Paulo, mas em todo o País, com repercussões na qualidade da assistência à saúde a ser oferecida à comunidades brasileira. Para a Universidade de São Paulo representa enriquecimento, pois suas Unidades de Enfermagem passarão a exercer liderança, em nível de ensino, na área de conhecimento ainda carente de recursos humanos destinados ao preparo do pessoal indisponível à melhora das condições sanitárias do Brasil.

Aproveitamos o ensejo para renovar a Vossa Excelência os nossos protestos de elevada estima e distinta consideração" (Universidade de São Paulo)(a)

À época de sua criação, a composição do quadro de docentes e orientadores envolveu, além dos docentes das duas Escolas de Enfermagem, professores de outras unidades da USP, sendo assim configurado, em sua versão original:

1 - Quadro de orientadores:

- 55 professores orientadores, vinculados às Escolas de Enfermagem e a outras unidades da USP;

2 - Elenco de disciplinas:

Composto pelas disciplinas dos mestrados das duas Unidades e outras especificamente criadas para o "Interunidades", distribuídas da seguinte forma:
A trajetória histórica do programa interunidades de doutoramento em enfermagem e sua contribuição para a enfermagem brasileira
(a)Fonte: arquivos das seções de Pós-graduação da EEUSP/ EERPUSP

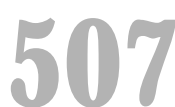

Rev Esc Enferm USP 2005; 39(Esp.):506-14. 
Maria G. C.Ferriani

Isília Aparecida Silva Emília C. de Carvalho

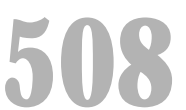

Rev Esc Enferm USP 2005; 39(Esp.):506-14.
- 2 disciplinas obrigatórias para alunos do “Interunidades": ENC 820 - Análise Crítica da Evolução da Assistência de Enfermagem e ENO 892 Problemática de Enfermagem;

- 37 disciplinas optativas;

- 28 disciplinas optativas de área complementar.

As unidades responsáveis pelo oferecimento de disciplinas, além da Escola de Enfermagem e Escola de Enfermagem de Ribeirão Preto, eram: Escola de Comunicação e Artes, Faculdade de Medicina, Faculdade de Saúde Pública, Instituto de Ciências Biomédicas, Instituto de Física, Instituto de Matemática e Estatística, Instituto de Psicologia, Faculdade de Filosofia, Ciências e Letras de Ribeirão Preto, Faculdades de Farmácia e Odontologia de Ribeirão Preto, Faculdade de Medicina de Ribeirão Preto.

O planejamento das disciplinas pretendia a inclusão de assuntos amplos de todas as áreas e que pudessem ser abordados sob diferentes ângulos e ao mesmo tempo sem serem esgotados, porém, transparecia uma forte influência do modelo biológico e funcionalista, com enfoque na metodologia positivista. Essa característica inicial do Programa é perfeitamente compreensível se for observado que a maioria dos docentes e orientadores, externos às unidades, eram provenientes de Departamentos das unidades biomédicas.

As Linhas de Pesquisa adotadas para o Programa foram emanadas dos Programas de Pós-Graduação já existentes nas duas Escolas.

O Programa Interunidades de Doutoramento em Enfermagem foi aprovado pela Câmara de Pós-Graduação da Universidade de São Paulo em 25 de maio de 1981, e a Resolução 2.204 de 9-6-81 correspondente foi publicada no Diário Oficial do Estado de São Paulo aos 10 de junho do mesmo ano, conforme se segue:

Artigo $1^{\circ}$ - O Programa de Doutorado em Enfermagem na Universidade de São Paulo será organizado e oferecido, em ação conjunta, pelas Escolas de Enfermagem dos "Campi" de São Paulo e de Ribeirão Preto.

Artigo $2^{\circ}$ - Além das normas gerais estabelecidas na presente Resolução, no Regimento Geral e na Portaria GR-885/69, o Programa de Doutorado em Enfermagem se regerá, no que couber, pelo Regulamento dos Programas de Pós-Graduação da Escola de Enfermagem de São Paulo, baixado pela Resolução no 2193 de 28 de maio de 1981.

Artigo $3^{\circ}$ - A coordenação do Programa será exercida por uma Comissão Interunidades, constituída por cinco docentes e um representante do corpo discente.
$\S 1^{\circ}$ - Os membros docentes, portadores pelo menos do título de Doutor, pertencentes três a uma Unidade e dois a outra, com alternância desta proporção a cada mandato, serão indicados juntamente com os correspondentes suplentes, pelos Diretores das respectivas Escolas de enfermagem e terão mandato de dois anos, permitida a recondução.

$\S 2^{\circ}$ - O representante discente, estudante de pós-graduação regularmente matriculado no Programa de Doutorado, não vinculado ao corpo docente da Universidade, será eleito por seus pares, com mandato de um ano, não renovável.

$\S 3^{\circ}$ - A composição da Comissão Interunidades deverá ser aprovada pela CPGr.

$\S 4^{\circ}$ - A comissão elegerá, dentre seus membros docentes, um presidente e um vice-presidente, com mandato de dois anos, vedada a recondução sucessiva.

Artigo $4^{\circ}$ - À Comissão Interunidades competem, no que couber, as atribuições da CPG previstas no artigo $3^{\circ}$ do Regulamento dos Programas de Pós-Graduação da Escola d Enfermagem.

Artigo $5^{\circ}$ - A Comissão Interunidades designará dois docentes, um de cada Unidade, portadores pelo menos do título de Doutor, para atuarem como Coordenadores e servirem de elementos de ligação entre a Comissão e os demais integrantes do Programa.

Artigo $6^{\circ}$ - Caberá à Comissão Interunidades indicar, à Congregação competente, os membros efetivos e suplentes das Comissões Julgadoras de teses, devendo dois dos membros e respectivos suplentes serem estranhos ao Departamento a que estiver vinculado o orientador.

$\S 1^{\circ}$ - A composição das Comissões Julgadoras referida neste artigo será aprovada pela Congregação da Unidade integrante do Programa de Doutorado à qual estiver vinculado o orientador.

$\S 2^{\circ}$ - Para fins exclusivos do previsto no parágrafo anterior, caberá a Comissão Interunidades indicar a vinculação do orientador que, embora integrante do Programa, não pertença funcionalmente a nenhuma das duas Unidades.

Artigo $7^{\circ}$ - O trabalho de secretaria do Programa de Doutorado será centralizado na Escola de Enfermagem.

Artigo $8^{\circ}$ - $O$ presente Regulamento entrará em vigor na data de sua publicação. Proc. RUSP $n^{\circ}$ 19249/81.

São Paulo(1)

Instalava-se, então, o primeiro Doutorado em Enfermagem do Brasil e da América Latina.

Em 5 de junho de 1986, o Programa foi credenciado pelo Conselho Federal de Educação, confor- 
me Parecer n. ${ }^{\circ}$ 363/86. Em 1984, o regulamento do Programa sofreu modificações, passando a ter a indicação de seus membros feita e aprovada pela Congregação das Unidades as quais pertence, entre outras, em especial no que concerne à indicação e aprovação da composição de bancas de qualificação e de defesa de tese.

A coordenação do Programa é exercida pela Comissão Interunidades de Pós-Graduação, que está subordinada à Congregação da EE e EERP, e atualmente sua composição é feita por três docentes de cada uma das Escolas de Enfermagem e por um representante discente. A presidência da Comissão Interunidades de Pós-Graduação alterna-se a cada dois anos entre os docentes da EE e da EERP, o mesmo acontecendo com a vice-presidência.

Ao longo de sua história, os docentes que cumpriram esse papel foram:

\section{1-1983}

Presidenta: Prof. ${ }^{a}$ Dr. ${ }^{a}$ Evalda Cançado Arantes - EE Vice-Presidenta: Prof. ${ }^{a}$ Dr. ${ }^{a}$ Maria Helena Machado -EERP-USP

1983-1985

Presidenta: Prof. ${ }^{a}$ Dr. ${ }^{a}$ Nilza Tereza Rotter Pelá - EERP Vice-Presidenta: Prof. ${ }^{a}$ Dr. ${ }^{a}$ Evalda Cançado Arantes $-\mathrm{EE}$

1985-1987

Presidenta: Prof. ${ }^{a}$ Dr. ${ }^{a}$. Yoriko Kamiyama - EE

Vice-Presidenta: Prof. ${ }^{a}$ Dr. ${ }^{a}$. Nilza Tereza Rotter Pelá -EERP-USP

\section{7-1989}

Presidenta: Prof. ${ }^{a}$ Dr. ${ }^{a}$ Maria Auxiliadora Trevizan EERP

Vice-Presidenta: Prof. ${ }^{a}$ Dr. ${ }^{a}$ Nara Sena de Paula - EE

1989-1991

Presidenta: Prof. ${ }^{a}$ Dr. ${ }^{\text {a }}$ Tâmara Iwanow Cianciarullo $-\mathrm{EE}$

Vice-Presidenta: Prof. ${ }^{a}$ Dr. ${ }^{a}$ Emília Campos de Carvalho - EERP

\section{1-1993}

Presidenta: Prof. ${ }^{a}$ Dr. ${ }^{a}$ Emília Campos de Carvalho EERP

Vice-Presidenta: Prof. ${ }^{a}$ Dr. ${ }^{a}$ Maguida Costa StefanelliEE

\section{3-1995}

Presidenta: Prof. ${ }^{a}$ Dr. ${ }^{a}$ Paulina Kurcgant - EE Vice-Presidenta: Prof. ${ }^{\text {a }}$ Dr. ${ }^{\text {a Semiramis Melani Melo }}$ Rocha-EERP
1995-1997

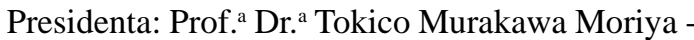
EERP

Vice-Presidenta: Prof. ${ }^{a}$ Dr. ${ }^{a}$ Emiko Yoshikawa Egry $\mathrm{EE}$

1997-1999

Presidenta: Prof. ${ }^{a}$ Dr. ${ }^{a}$ Emiko Yoshikawa Egry - EE

Vice-Presidenta: Prof. a Dr. . Maria das Graças Bonfim de Carvalho - EERP

1999-2001

Presidenta: Prof. ${ }^{a}$ Dr. ${ }^{a}$ Maria das Graças Bonfim de Carvalho - EERP

Vice-Presidenta: Prof. ${ }^{\text {a } D r . ~}{ }^{a}$ Isília Aparecida Silva $\mathrm{EE}$

2001-2003

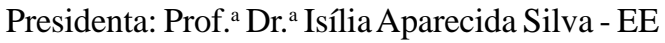

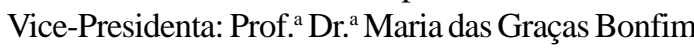
de Carvalho - EERP

\section{3-2005}

Presidenta: Prof. ${ }^{a}$ Dr. ${ }^{a}$ Maria das Graças Bonfim de Carvalho - EERP

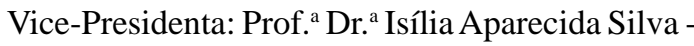
$\mathrm{EE}$

O primeiro processo seletivo de alunos realizou-se em outubro de 1981, admitindo-se 12 enfermeiros. Das vagas abertas apenas duas destinaram-se à demanda externa, visto que havia necessidade premente de titular os docentes das Unidades mantenedoras do Programa, em face da pressão da Reforma Universitária, introduzida pela Lei 5.540/ 68. A primeira turma iniciou suas atividades a partir de março de 1982.

Durante muitos anos, até por volta do início da década de 1990, a demanda principal de candidatos era das próprias Unidades responsáveis pelo curso, seguida de professores de outras Escolas de Enfermagem do Brasil.

A progressiva titulação dos docentes da casa reduzia, gradativamente, a demanda reprimida nas Escolas de Enfermagem da USP, permitindo a ampliação de vagas para enfermeiros docentes e assistenciais de outras instituições. Em 1986, surgiu o segundo doutorado em enfermagem pela iniciativa do Departamento de Enfermagem da então Escola Paulista de Medicina, hoje, Universidade Federal de São Paulo, tendo como área de concentração a “Enfermagem Materno-Infantil”, abrindo, dessa forma, mais uma opção para os enfermeiros em busca de formação para a pesquisa.
A trajetória histórica do programa interunidades de doutoramento em enfermagem e sua contribuição para a enfermagem brasileira 
Maria G. C.Ferriani

Isília Aparecida Silva

Emília C. de Carvalho
Na década de 1990, houve uma franca expansão dos cursos de doutorado em enfermagem no País. O aumento da oferta em diferentes regiões geográficas brasileiras favoreceu o atendimento de um maior número de profissionais. Hoje, a demanda do Programa Interunidades de Doutoramento em Enfermagem, ao contrário do que ocorria em sua gênese, caracteriza-se pela diversidade de candidatos oriundos de diferentes Estados do País, diferentes instituições de ensino e de serviços.

Ao longo de sua trajetória, o Programa Interunidades recebeu conceito máximo nas avaliações realizadas pela CAPES - Coordenação de Aperfeiçoamento de Pessoal de Nível Superior, até 1995, e no triênio 1998/2000, teve sua nota diminuída, caindo para a nota quatro. Esse fato exigiu esforço conjunto de seus docentes, o que resultou em recuperação da nota cinco para o triênio 2001/2003, conforme Quadro 1.

Quadro 1 - Conceitos e notas atribuídas pela CAPES, na avaliação do Programa Interunidades de Doutoramento em Enfermagem, no período de 1983 a 2000.

\begin{tabular}{|c|c|}
\hline PERÍODO & CONCEITO-NOTA \\
\hline $1983-1984$ & $\mathrm{~A}$ \\
$1985-1986$ & $\mathrm{~A}$ \\
$1988-1989$ & $\mathrm{~A}$ \\
$1990-1991$ & $\mathrm{~A}$ \\
$1992-1993$ & $\mathrm{~A}$ \\
$1994-1995$ & $\mathrm{~A}$ \\
$1996-1997$ & 5 \\
$1998-2000$ & 4 \\
$2001-2003$ & 5 \\
\hline
\end{tabular}

A matriz de organização do Programa Interunidades foi modelo para a criação de muitos Programas de Pós-Graduação da Enfermagem no Brasil. No entanto, a mudança de paradigma de avaliação dos Programas de Pós-Graduação, pela CAPES, adotada progressivamente no final da década de 1990, fez com que, em 1998, os conceitos e notas recebidos dos dois últimos triênios de avaliação fossem rebaixados, indicando o ponto de inflexão, percebido pela sua coordenação para promover uma nova configuração do Programa.
Tomando como base os aspectos salientados pela avaliação CAPES 1998/2000, o Programa Interunidades de Doutoramento em Enfermagem iniciou, nesse período, a Reformulação do Programa, uma vez que a CAPES manifestou a necessidade de caracterização de um perfil epistemológico específico que o diferenciasse dos demais Programas de Doutorado das duas Unidades e do restante do País, e que se refletisse nas linhas e projetos de pesquisa e produção docente.

Os docentes das duas Unidades credenciados no Programa foram consultados quanto ao interesse em permanecer no Programa, orientando e/ou ministrando disciplinas, resultando em um grupo considerável de docentes e alunos interessados pela manutenção do Programa, manifestado também pelas Diretorias das duas Unidades.

Com o objetivo de identificar a vocação, a missão e o direcionamento de novas tendências do Programa Interunidades de Doutoramento foram realizados desde 1998 um total de nove Fóruns até o presente, diante de avaliações sucessivas da CAPES e necessidades percebidas de reformulação do programa, procurando atender a um novo cenário da Pós-Graduação de Enfermagem no Brasil.

Assim, com tal finalidade, docentes e discentes do Programa das duas Unidades e seus coordenadores, durante esse período, definiram uma estrutura do Programa Interunidades, cujo perfil vem sendo aprimorado ao longo desses quatro anos.

Esse processo dinâmico, e que se configura mediante avaliações sistemáticas e busca do atendimento da demanda por programas de pós-graduação com consistência em atividades de pesquisa.

Esse processo resultou na definição de um novo desenho para o Interunidades, cuja formatação é sustentada pela nucleação central Bases Teóricas e Filosóficas do Saber e da Prática de Enfermagem, de onde convergem as disciplinas e se articulam as linhas e projetos de pesquisa, configurando as áreas de produção científica ${ }^{(2)}$

Seis linhas de pesquisa constituem a nucleação central, sustentadas por 16 disciplinas, conforme figura a seguir: 


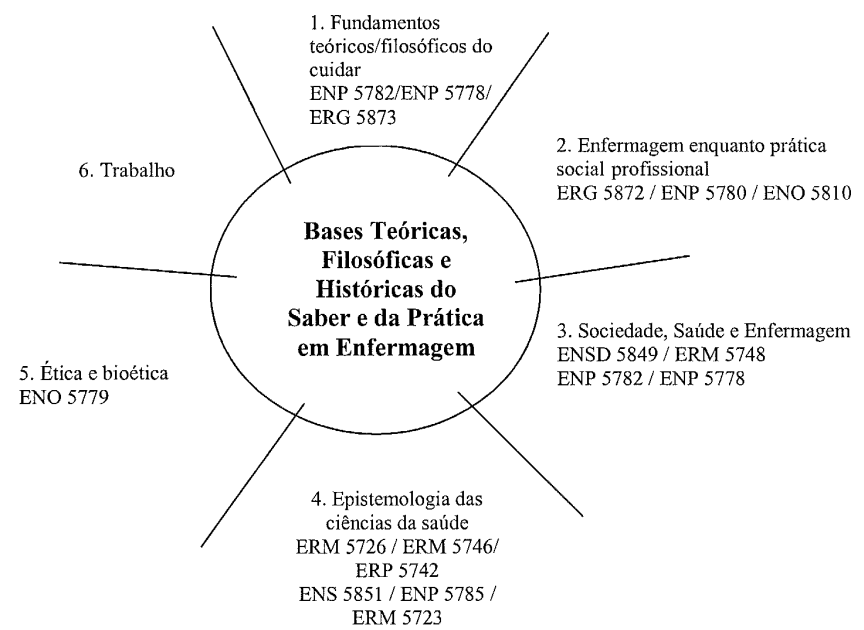

Fig. 1 - Nucleação central e linhas de pesquisa do Programa Interunidades de doutoramento em Enfermagem. São Paulo, 2004.

Os fóruns sucessivos têm servido para o aprimoramento da estrutura do Programa, resultando ainda, no final desse período, na reformulação das linhas de pesquisa e suas ementas. Buscaram-se o delineamento e o fortalecimento de linhas que já se mostravam consolidadas, elencadas a seguir com as respectivas disciplinas:

- Enfermagem enquanto prática Social/ Profissional

Versa sobre a análise histórica, social e filosófica das práticas de saúde e de enfermagem e suas relações com as políticas públicas em nível nacional e internacional.

ERG 5872 - Análise crítica da evolução da assistência de enfermagem

ENP 5780 - As políticas de saúde e a construção da prática de enfermagem

ENO 5810 - Estudos avançados em gerencia-mento em enfermagem

\section{- Epistemologia das ciências da saúde}

Estudos analíticos sobre a construção da ciência em saúde e em enfermagem sobre os referenciais teóricos e metodológicos, princípios e estratégias de análise de dados em pesquisa qualitativa e sua relação e contribuição para a prática de investigação em enfermagem.

ERM 5726 - A construção do conhecimento em saúde I

ERM 5746 - Abordagem qualitativa em pesquisa social na saúde

ERP 5742 - Pesquisa fenomenológica: questões fundamentais

ENS 5851 - Gênero, saúde e enfermagem: uma abordagem epistemológica

ENP 5785 - Elementos de análise de discurso aplicados à enfermagem
ERM 5723 - Epistemologia e teoria feminista: aplicação em projetos de pesquisa

ENP 5782 - Obtenção e análise de dados qualitativos

\section{- Fundamentos teóricos e filosóficos do cuidar}

Engloba pesquisas que têm a finalidade de aprofundar os conhecimentos relativos às várias nuances do processos saúde-doença e a construção social da doença, relacionados com a ética e bioética, com a cultura, com o corpo e seu processo histórico e cultural.

ENP 5782 - O processo saúde doença no curso da vida

ENP 5778 - O processo saúde doença na perspectiva cultural

ENP 5873-Sistema de classificação em Enfermagem ENO 5779-Bioética em foco: uma abordagem filosófica e conceitual

\section{- Sociedade, saúde e Enfermagem}

Compreende estudos sobre a articulação teórico-conceitual da estruturação das sociedades e as correspondentes maneiras de produção da saúde e de processos de desenvolvimento da enfermagem enquanto prática social.

ENS 5849 - Gênero, saúde e enfermagem: uma abordagem conceitual

ERM 5748 - Promoção de saúde no contexto do desenvolvimento humano

ENP 5782 - O processo saúde doença no curso da vida

ENP 5778 - O processo saúde doença na perspectiva cultural

Dessa forma, o Programa está constituído, atualmente, por quatro linhas de pesquisas. Considerando-se que, o processo/atividade de pesquisa
A trajetória histórica do programa interunidades de doutoramento em enfermagem e sua contribuição para a enfermagem brasileira 
Maria G. C.Ferriani

Isília Aparecida Silva

Emília C. de Carvalho

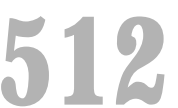

Rev Esc Enferm USP 2005; 39(Esp.):506-14. e ensino, é um fenômeno dinâmico, as linhas emergentes do Programa deverão acumular atividades de pesquisa e produção compatíveis com a densidade de consolidação científica vertical, para serem agregadas à nucleação central do programa. Nessa condição, estão ainda como emergentes, as linhas Trabalho e Ética e Bioética, que deverão ser ativadas em 2005.

No que concerne à seleção dos candidatos, o Programa passou a exigir melhor formação do aluno, também em língua estrangeira, objetivando aumentar as possibilidades de absorver um alunado mais competitivo e preparado para expandir suas atividades acadêmicas em instituições de excelência no exterior.

Ressaltamos, ainda, que dentre as prioridades estabelecidas pelo Programa, a produção científica dos docentes e discentes e o fluxo de titulação dos doutorandos têm merecido especial atenção. Desde sua reformulação, estão sendo implementadas novas estratégias para conclusão e titulação abreviada, bem como o incentivo para a produção sistemática do aluno em parceria com o orientador, no decorrer de sua formação. Entre essas, faz parte a inclusão do aluno em programas e grupos de pesquisa de seu orientador, a realização de seminários avançados que visam a atender às necessidades do alunado para melhor definir o desenho metodológico e temático dos projetos de tese, intensificar e propiciar melhor intercâmbio de docentes e discentes das duas unidades em que o Programa se desenvolve, por meio de disciplinas conjuntas e utilização de estratégias e tecnologia de informação aplicadas ao ensino. Ainda deve-se dar continuidade ao incentivo da participação do corpo docente e discente em eventos científicos para divulgação de suas pesquisas, tendo como uma das estratégias a ajuda de custo para pagamento de despesas.

Também, mediante avaliação positiva, de alunos e discentes, mantém-se em uma das Unidades, onde o Programa se desenvolve, a estratégia de discussões de projetos dos alunos do Programa durante a disciplina ERM 5746 - “Abordagem Qualitativa em Pesquisa Social na Saúde”, possibilitando a vinda de professores convidados especialistas nessa metodologia, bem como propiciando a participação de alunos de ambas as unidades sedes do Programa, objetivando maior intercâmbio. Tem-se procurado incentivar os alunos das duas Unidades onde o Programa se realiza, a freqüentar disciplinas que são oferecidas não só pelo Campus Ribeirão Preto como o de São Paulo.

No que se refere aos créditos, os alunos têm flexibilidade para seu cumprimento dentro do elen- co de disciplinas do Programa e em outros cursos dentro ou fora das Unidades sedes, desde que cumpram 40\% em disciplinas do "Interunidades".

A reformulação realizada no Programa, desde 1998, além de influir na proposta pedagógica e epistemológica do "Interunidades", implicou na definição de seu quadro docente/orientadores, mantendo-se credenciamento daqueles professores que tivessem, efetivamente, vínculo temático e metodológico à nucleação central e linhas de pesquisa do Programa. A inclusão de praticamente todos os docentes de ambas as Unidades, EE e EERP, no quadro do Programa, elevou numericamente o número de orientadores chegando a 65 em 1997, também com o ápice de corpo discente. Dos 55 professores credenciados no início de sua implantação, o Programa conta hoje com 23 docentes, sendo todos do quadro próprio das Unidades sede do "Interunidades", o que demonstra sua independência de recursos humanos externos para cumprir sua vocação. A tendência que se observa é de manutenção desse número de docentes, seja pela inclusão espontânea de novos orientadores pela sua aderência ao perfil e proposta do Programa, seja pela finalização de atividades de outros, que ainda estão vinculados ao Programa, por compromissos assumidos no período de reformulação que ainda estão pendentes.

Quadro 2 - Evolução do número de alunos matriculados, jan./dez./ ano no Programa Interunidades de Doutoramento em Enfermagem, no período de 1981 a 2004.

\begin{tabular}{|c|c|}
\hline ANO & N. DE ALUNOS \\
\hline 1981 & 12 \\
1982 & 24 \\
1983 & 35 \\
1984 & 49 \\
1985 & 42 \\
1986 & 47 \\
1987 & 38 \\
1988 & 48 \\
1989 & 56 \\
1990 & 65 \\
1991 & 64 \\
1992 & 72 \\
1993 & 72 \\
1994 & 78 \\
1995 & 83 \\
1996 & 92 \\
1997 & 93 \\
1998 & 95 \\
1999 & 72 \\
2000 & 69 \\
2001 & 77 \\
2002 & 59 \\
2003 & 60 \\
2004 & 48 \\
\hline
\end{tabular}

Fonte: Sistema Fênix. 
A procura pelo Programa tem assegurado uma freqüência regular, e como já mencionado, os alunos são originários dos mais diferentes Estados brasileiros, mantendo a demanda por docentes de instituições de ensino, porém, havendo o ingresso de muitos profissionais da área assistencial, implicando na mudança de perfil do alunado e nos interesses no desenvolvimento de pesquisas, obrigando o Programa a buscar novas tendências metodológicas e pedagógicas.

O Programa Interunidades de Doutoramento em Enfermagem vem colaborando com o avanço da Investigação em Enfermagem, além da demanda espontânea de alunos de diversos locais do País, por meio de convênios, o Programa titulou grupos de docentes da Universidade Federal da Paraíba, Universidade Federal da Bahia e Universidade Federal do Rio Grande do Norte e mantém entendimentos com a Universidade Federal de Sergipe, para o mesmo propósito. No momento, é partícipe de projetos conjuntos Programas de Qualificação Institucional, PQI, com Universidade de Pernambuco (UPE) (exclusivo do Inter), Faculdade de Medicina de São José do Rio Preto - FAMERP (Programas: Interunidades, Enfermagem Fundamental e Enfermagem em Saúde Pública), Escola de Farmácia e Odontologia de Alfenas - EFOA (Programas Interunidades, Enfermagem Fundamental e Enfermagem em Saúde Pública).

O Programa Interunidades tem formado pesquisadores para diversas Universidades brasileiras e da América do Sul, que formam e lideram células de pesquisa, dando origem a novos programas de pósgraduação. Para ilustrar, dentre os atuais Presidentes de Comissão de Pós-graduação e coordenadores dos 24 programas de pós-graduação do Brasil, pelo menos 16 foram titulados pelo Programa Interunidades de Doutoramento em Enfermagem, sendo que esta proporção tem se mantido nas últimas duas décadas.

O Programa formou, também, quatro doutores do Departamento de Enfermagem da Faculdade de Medicina da Universidade de Concepción, responsáveis, atualmente, pela instalação do primeiro doutorado em Enfermagem no Chile. Contribuiu, ainda, com a titulação de docentes da Universidade de Los Llanos Orientales, Colômbia e de Trás-os-Montes, Portugal.

Embora os números não sejam capazes de expressar o potencial qualitativo do que tem representado o Programa Interunidades para a Enfermagem Brasileira, é possível vislumbrar seu impacto quantitativo nos quase trezentos doutores egressos nos seus 22 anos de existência, conforme mostra o Quadro 3.
Quadro 3 - Títulos de pós-graduação outorgados pelo Programa Interunidades de Doutoramento em Enfermagem, até dezembro de 2004.

\begin{tabular}{|c|c|}
\hline ANO & N. DE TÍTULOS \\
\hline 1983 & 01 \\
1984 & 04 \\
1985 & 06 \\
1986 & 06 \\
1987 & 10 \\
1988 & 09 \\
1989 & 03 \\
1990 & 14 \\
1991 & 13 \\
1992 & 07 \\
1993 & 17 \\
1994 & 13 \\
1995 & 19 \\
1996 & 11 \\
1998 & 21 \\
1999 & 16 \\
2000 & 39 \\
2001 & 20 \\
2002 & 22 \\
2003 & 15 \\
2004 & 15 \\
\hline
\end{tabular}

Fonte: Arquivos das Seções de Pós-graduação da EE e da EERP

Outros níveis de impacto do doutorado são verificados na gestão das próprias Universidades, pois a titulação permite o ingresso efetivo na carreira docente e impulsiona a assunção de cargos e funções em diferentes escalões administrativos. Nesse aspecto há egressos do Interunidades, nas mais diversas funções administrativas de relevância, assumindo a liderança não só na criação e coordenação de programas de graduação e pós-graduação, mas exercendo funções como diretores de Faculdades, Chefes de Departamentos, representantes em órgãos de fomento, e até Reitora de Universidade Federal.

Além da influência acadêmica e administrativa, a assistência de Enfermagem também se beneficia, à medida que egressos do Programa atuam em Instituições de saúde diversas, podendo transferir o conhecimento adquirido para a prática assistencial, imprimindo também a consolidação da pesquisa como atividade do enfermeiro.

A dinâmica da ciência, o surgimento de nova oportunidade para doutoramento no Brasil, permitiram ao Programa Interunidades de Doutoramento em Enfermagem lançar um olhar crítico e maduro sobre si mesmo, calçado na experiência acumulada e em sintonia com o cenário científico atual.

O Programa Interunidades surge no terceiro milênio totalmente reformulado, resultado de intensas discussões realizadas entre 1998 e 2000, com metas de internacionalização para o próximo lustro.
A trajetória histórica do programa interunidades de doutoramento em enfermagem e sua contribuição para a enfermagem brasileira

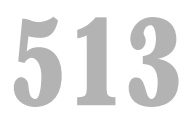

Rev Esc Enferm USP 2005; 39(Esp.):506-14. 
Maria G. C.Ferriani

Isília Aparecida Silva

Emília C. de Carvalho
A determinação e a chama de vanguarda que nutria o espírito das seis enfermeiras idealizadoras deste Programa pioneiro: Professoras Wanda, Amália, Evalda, Maria Aparecida, Maria Helena e Nilza se fazem sentir também, neste momento, em que o curso assume nova estrutura, com desafios de internacionalização a vencer, coexistindo em sinergia com outros programas de Pós-graduação já consolidados na EE e EERP, pelo avanço da Enfermagem.

\section{REFERÊNCIAS}

(1) São Paulo.(Estado). Resolução n. ${ }^{\circ}$ 2204, de 09 de junho de 1981. Aprova as normas que regulam o Programa de Doutoramento em Enfermagem. Diário Oficial do Estado, São Paulo, 10 de jun. 1981. Seção 1, p. 18-9.
(2) Escola de Enfermagem USP, Escola de Enfermagem Ribeirão Preto da USP. Relatório Coleta CAPES 2003 do Programa Interunidades de Doutoramento em Enfermagem. São Paulo; 2003. (Caderno: Programa 23). 